\title{
Neuronal ceroid lipofuscinosis
}

INSERM

\section{Source}

INSERM. (1999). Orphanet: an online rare disease and orphan drug data base. Neuronal ceroid lipofuscinosis. ORPHA:216

Neuronal ceroid lipofuscinoses (NCLS) are a group of inherited progressive degenerative brain diseases characterized clinically by a decline of mental and other capacities, epilepsy, and vision loss through retinal degeneration, and histopathologically by intracellular accumulation of an autofluorescent material, ceroid lipofuscin, in the neuronal cells in the brain and in the retina. 